\title{
Recent advances in understanding \\ the molecular basis of group B \\ Streptococcus virulence - \\ CORRIGENDUM
}

\section{Heather C. Maisey, Kelly S. Doran and Victor Nizet}

doi: 10.1017/S1462399408000811, Published by Cambridge University Press, 22 September 2008

There was an error in the text on page 6 and in Figure 2 of the review by H.C. Maisey et al. (Ref. 1). The complement protein that binds to BibA is C4bp rather than C3bp.

\section{Reference}

1 Maisey, H.C., Doran, K.S. and Nizet, V. (2008) Recent advances in understanding the molecular basis of group B Streptococcus virulence. Expert Rev. Mol. Med. 10, e27 